\title{
Chebyshev polynomials for solving two dimensional linear and nonlinear integral equations of the second kind
}

\author{
ZAKIEH AVAZZADEH* and MOHAMMAD HEYDARI \\ Faculty of Science, Islamic Azad University, Yazd Branch, Yazd, Iran \\ E-mails: z.avazzadeh@yahoo.com/m.heydari85@gmail.com
}

\begin{abstract}
In this paper, an efficient method is presented for solving two dimensional Fredholm and Volterra integral equations of the second kind. Chebyshev polynomials are applied to approximate a solution for these integral equations. This method transforms the integral equation to algebraic equations with unknown Chebyshev coefficients. The high accuracy of this method is verified through some numerical examples.
\end{abstract}

Mathematical subject classification: $65 \mathrm{R} 20,41 \mathrm{~A} 50,41 \mathrm{~A} 55,65 \mathrm{M} 70$.

Key words: Chebyshev polynomials, two dimensional integral equations, collocation method.

\section{Introduction}

Two dimensional integral equations provide an important tool for modeling a numerous problems in engineering and science $[2,12]$. These equations appear in electromagnetic and electrodynamic, elasticity and dynamic contact, heat and mass transfer, fluid mechanic, acoustic, chemical and electrochemical process, molecular physics, population, medicine and in many other fields $[6,7,8,14$, $18,20]$.

The Nystrom method [10] and collocation method [3,21] are the most important approaches of the numerical solution of these integral equations. Recently,

\#CAM-326/11. Received: 25/I/11. Accepted: 01/VII/11.

*Corresponding author. 
some new methods such as differential transform method are applied for solving two dimensional linear and nonlinear Volterra integral equations [1, 5, 19].

In this work, we will apply the Chebyshev polynomials for solving two dimensional integral equations of second kind. The use of the Chebyshev series for the numerical solution of linear integral equations has previously been discussed in [17] and references therein. The privilege of the method is simplicity and spectral accuracy $[4,11]$. The examples confirm that the method is considerably fast and highly accurate as sometimes lead to exact solution. Also, this method lead to continuous solution covering all the domain.

The paper is organized in the following way. In Section 2 the famous Chebyshev polynomials and its application are introduced [16]. In Section 3 the method of solution of the linear two dimensional integral equation of second kind is described. In Section 4 we will try to generalize this method for nonlinear cases. In Section 5 some examples are chosen to show the ability and high accuracy of the method.

\section{Chebyshev polynomials}

Definition 1. If $t=\cos \theta(0 \leqslant \theta \leqslant \pi)$, the function

$$
T_{n}(t)=\cos (n \theta)=\cos (n \arccos t),
$$

is a polynomial of $t$ of degree $n(n=0,1,2, \ldots) . T_{n}$ is called the Chebyshev polynomial of degree $n$ [16]. When $\theta$ increase from 0 to $\pi, t$ decrease from 1 to -1 . Then the interval $[-1,1]$ is domain of definition of $T_{n}(t)$. It satisfies the orthogonality condition

$$
\int_{-1}^{1} \frac{T_{n}(x) T_{m}(x)}{\sqrt{1-x^{2}}}= \begin{cases}0, & n \neq m, \\ \frac{\pi}{2}, & n=m \neq 0, \\ \pi, & n=m=0\end{cases}
$$

Remark 1 (Chebyshev series expansion). Let be $g(x)$ a function on $[a, b]$. For a given arbitrary natural number $M$, Chebyshev series expansion of $g(x)$ have the form

$$
g(x) \simeq \sum_{k=0}^{M} a_{k} T_{k}\left(\frac{2}{b-a} x-\frac{b+a}{b-a}\right), \quad x \in[a, b],
$$


where

$$
a_{k}=\frac{2}{\pi c_{k}} \int_{-1}^{1} \frac{g\left(\frac{b-a}{2} x+\frac{b+a}{2}\right) T_{k}(x)}{\sqrt{1-x^{2}}} d x, \quad k=1,2, \cdots, M .
$$

and

$$
c_{k}= \begin{cases}2, & k=0 \\ 1, & k \geq 1\end{cases}
$$

Definition 2. Suppose $f(x, t)$ be a continuous function on $[-1,1] \times[-1,1]$. For a given natural number $N$, we set

$$
f(x, t) \approx \sum_{i=0}^{N} \sum_{j=0}^{N} a_{i j} T_{i}(x) T_{j}(t), \quad(x, t) \in[-1,1] \times[-1,1],
$$

where

$$
a_{i j}=\frac{\left\langle T_{i}(x),\left\langle f(x, t), T_{j}(t)\right\rangle\right\rangle}{\left\langle T_{i}(x), T_{i}(x)\right\rangle \cdot\left\langle T_{j}(t), T_{j}(t)\right\rangle},
$$

and $\langle\cdot, \cdot\rangle$ denotes the inner product in function space $L^{2}([-1,1] \times[-1,1])$.

Remark 2. This paper discusses using Chebyshev polynomials of the first kind to approximate functions on finite interval, that is, on the interval $[-1,1]$. Practically, other polynomials, which are orthogonal on finite interval, can also be applied for approximating functions. But the partial sums of a first-kind Chebyshev expansion of a continuous function in $[-1,1]$ cover faster than the partial sums of an expansion in any other orthogonal polynomials [16].

\section{Solution of linear two dimensional integral equation}

Consider the two dimensional linear Fredholm and Volterra integral equations as follows

$$
\begin{gathered}
u(x, t)-\int_{-1}^{1} \int_{-1}^{1} k(x, t, y, z) u(y, z) d y d z=f(x, t) \\
(x, t) \in[-1,1] \times[-1,1]
\end{gathered}
$$


and

$$
\begin{gathered}
u(x, t)-\int_{-1}^{t} \int_{-1}^{x} k(x, t, y, z) u(y, z) d y d z=f(x, t), \\
(x, t) \in[-1,1] \times[-1,1],
\end{gathered}
$$

where $u(x, t)$ is an unknown scalar-valued function, $f(x, t)$ and $k(x, t, y, z)$ are continuous functions on $[-1,1]^{2}$ and $[-1,1]^{4}$ respectively. For the case which integration domain is $[a, b] \times[c, d]$, we can use suitable change of variable to obtain these intervals.

At first, we consider two dimensional linear Fredholm integral equations are defined in (4). Function $u(x, t)$ defined over $[-1,1] \times[-1,1]$ may be represented by Chebyshev series as

$$
u(x, t)=\sum_{i=0}^{\infty} \sum_{j=0}^{\infty} a_{i j} T_{i}(x) T_{j}(t), \quad(x, t) \in[-1,1] \times[-1,1] .
$$

If the infinite series in (6) is truncated, then (6) can be written as

$$
u(x, t) \approx u_{N}(x, t)=\sum_{i=0}^{N} \sum_{j=0}^{N} a_{i j} T_{i}(x) T_{j}(t),
$$

where $N$ is any natural number. The method of collocation solves the (4) using the approximation (7) through the equations

$$
\begin{gathered}
R_{N}\left(x_{r}, t_{s}\right)=u_{N}\left(x_{r}, t_{s}\right)-\int_{-1}^{1} \int_{-1}^{1} k\left(x_{r}, t_{s}, y, z\right) u_{N}(y, z) d y d z \\
-f\left(x_{r}, t_{s}\right)=0,
\end{gathered}
$$

for Gauss-Chebyshev-Lobatto as collocation points [16]

$$
\begin{cases}x_{r}=\cos \left(\frac{r \pi}{N}\right), & r=0,1, \ldots, N, \\ t_{s}=\cos \left(\frac{s \pi}{N}\right), & s=0,1, \ldots, N .\end{cases}
$$

The interested reader can see more detail of collocation method in $[3,4,11]$.

Similarly, function $k\left(x_{r}, t_{s}, y, z\right)$ can be expressed as truncated Chebyshev series in the following form

$$
k\left(x_{r}, t_{s}, y, z\right) \approx k_{M}\left(x_{r}, t_{s}, y, z\right)=\sum_{p=0}^{M} \sum_{q=0}^{M} k_{p q}^{(r, s)} T_{p}(y) T_{q}(z),
$$


for any natural number $M$. From (3) we have

$$
k_{p q}^{(r, s)}=\frac{4}{\pi^{2} c_{p} c_{q}} \int_{-1}^{1} \int_{-1}^{1} \frac{k\left(x_{r}, t_{s}, y, z\right)}{\sqrt{1-y^{2}} \sqrt{1-z^{2}}} T_{p}(y) T_{q}(z) d y d z,
$$

where

$$
c_{p}= \begin{cases}2 & p=0 \\ 1 & p \geq 1\end{cases}
$$

By using Gauss-Chebyshev-Lobatto integration rule [13], for a given natural number $n$ we have

$$
k_{p q}^{(r, s)}=\frac{4}{n^{2} c_{p} c_{q}} \sum_{\xi=0}^{n} \sum_{\eta=0}^{\prime \prime} k\left(x_{r}, t_{s}, \cos \frac{\xi \pi}{n}, \cos \frac{\eta \pi}{n}\right) \cos \left(\frac{p \xi \pi}{n}\right) \cos \left(\frac{q \eta \pi}{n}\right),
$$

where double prime denotes that the first and the last terms are halved. Now, by substituting (7) and (10) into (8) we obtain

$$
\sum_{i=0}^{N} \sum_{j=0}^{N} a_{i j} \psi_{i j}^{r s}-f\left(x_{r}, t_{s}\right)=0 .
$$

where

$$
\psi_{i j}^{r s}=T_{i}\left(x_{r}\right) T_{j}\left(t_{s}\right)-\sum_{p=0}^{M} \sum_{q=0}^{M} k_{p q}^{(r, s)}\left(\int_{-1}^{1} \int_{-1}^{1} T_{i}(y) T_{j}(z) T_{p}(y) T_{q}(z) d y d z\right) .
$$

We define

$$
\begin{aligned}
b_{i j}^{(r, s)} & =T_{i}\left(x_{r}\right) T_{j}\left(t_{s}\right), \\
f_{r s} & =f\left(x_{r}, t_{s}\right),
\end{aligned}
$$

and

$$
w_{p q}^{(i, j)}=\left(\int_{-1}^{1} T_{i}(y) T_{p}(y) d y\right)\left(\int_{-1}^{1} T_{j}(z) T_{q}(z) d z\right) .
$$

But the Chebyshev polynomials are even for even degree and odd for odd degree. Hence,

$$
\int_{-1}^{1} T_{i}(x) T_{p}(x) d x=\left\{\begin{array}{cll}
\frac{1}{1-(i+p)^{2}}+\frac{1}{1-(i-p)^{2}} & i+p & \text { is even } \\
0 & i+p & \text { is odd. }
\end{array}\right.
$$


So, from (13), (14), (15) and (16) we can obtain the system of linear equations,

$$
\sum_{i=0}^{N} \sum_{j=0}^{N} a_{i j} \tilde{w}_{i j}^{(r, s)}=f_{r s}, \quad r, s=0,1,2, \ldots, N,
$$

where $\tilde{w}_{i j}^{(r, s)}$ is computed by the following relation

$$
\tilde{w}_{i j}^{(r, s)}=b_{i j}^{(r, s)}-\sum_{p=0}^{M} \sum_{q=0}^{M} k_{p q}^{(r, s)} w_{p q}^{(i, j)}, \quad i, j=0,1, \ldots, N .
$$

Clearly, the obtained system contains $(N+1)^{2}$ equations in the same number as unknowns. It can be solved by Newton's iteration method to obtain the value of $a_{i j}$ such that $i, j=0 \ldots N$.

For the Volterra case, this method is valid. We just change (16) with the following equation

$$
w_{p q}^{(i, j, r, s)}=\left(\int_{-1}^{x_{r}} T_{i}(y) T_{p}(y) d y\right)\left(\int_{-1}^{t_{s}} T_{j}(z) T_{q}(z) d z\right),
$$

so that, if let

$$
\tau_{i p}(x)=\int_{-1}^{x} T_{i}(y) T_{p}(y) d y
$$

we will have

$$
\tau_{i p}(x)=\frac{1}{4}\left\{\begin{array}{l}
2 x^{2}-2 \\
\frac{T_{i+p+1}(x)}{i+p+1}-\frac{T_{i+p-1}(x)}{i+p-1}-\frac{1}{i+p+1}+\frac{1}{i+p-1}+x^{2}-1 \\
\frac{T_{i+p+1}(x)}{i+p+1}+\frac{T_{1-i-p}(x)}{1-i-p}+\frac{T_{1+i-p}(x)}{1+i-p}+\frac{T_{1-i+p}(x)}{1-i+p} \\
\quad+\frac{2}{1-(i+p)^{2}}+\frac{2}{1-(i-p)^{2}} \\
\frac{T_{i+p+1}(x)}{i+p+1}+\frac{T_{1-i-p}(x)}{1-i-p}+\frac{T_{1+i-p}(x)}{1+i-p}+\frac{T_{1-i+p}(x)}{1-i+p} \\
-\frac{2}{1-(i+p)^{2}}-\frac{2}{1-(i-p)^{2}}
\end{array}\right.
$$

when respectively $i+p=1,|i-p|=1, i+p$ is even and $i+p$ is odd. 


\section{Solution of nonlinear two dimensional integral equation}

Consider the two dimensional nonlinear Fredholm and Volterra integral equations as follows

$$
\begin{gathered}
u(x, t)-\int_{-1}^{1} \int_{-1}^{1} k(x, t, y, z) F(u(y, z)) d y d z=f(x, t), \\
(x, t) \in[-1,1]^{2},
\end{gathered}
$$

and

$$
\begin{gathered}
u(x, t)-\int_{-1}^{t} \int_{-1}^{x} k(x, t, y, z) F(u(y, z)) d y d z=f(x, t), \\
(x, t) \in[-1,1]^{2},
\end{gathered}
$$

where $k(x, t, y, z)$ is continuous on $[-1,1]^{4}$, and $f(x, t)$ and $F(u(y, z))$ are continuous on $[-1,1]^{2}$. Again, for the case which integration domain is $[a, b] \times$ $[c, d]$, we can use suitable change of variable to obtain this intervals.

Before solving the above equations we exchange them with following equations. The other cases can be approximated in this form using Taylor extension. It reduce the related computation effectively.

$$
\begin{gathered}
u(x, t)-\int_{-1}^{1} \int_{-1}^{1} k(x, t, y, z)[u(y, z)]^{p} d y d z=f(x, t), \\
(x, t) \in[-1,1]^{2},
\end{gathered}
$$

and

$$
\begin{gathered}
(x, t)-\int_{-1}^{t} \int_{-1}^{x} k(x, t, y, z)[u(y, z)]^{p} d y d z=f(x, t), \\
(x, t) \in[-1,1]^{2},
\end{gathered}
$$

where $p$ is a positive integer number and $p \geq 2$. Correspondingly the linear case, by using (7) and (24) and considering collocation points we have

$$
u_{N}\left(x_{r}, t_{s}\right)-\int_{-1}^{1} \int_{-1}^{1} k\left(x_{r}, t_{s}, y, z\right)\left[u_{N}(y, z)\right]^{p} d y d z-f\left(x_{r}, t_{s}\right)=0 .
$$

Now, we replace (10) into above equation and if we let

$$
v_{p q}=\int_{-1}^{1} \int_{-1}^{1} T_{p}(y) T_{q}(z)\left[u_{N}(y, z)\right]^{p} d y d z,
$$


we have

$$
\begin{gathered}
\sum_{i=0}^{N} \sum_{j=0}^{N} a_{i j} T_{i}\left(x_{r}\right) T_{j}\left(t_{s}\right)-\sum_{p=0}^{M} \sum_{q=0}^{M} k_{p q}^{(r, s)} v_{p q}=f\left(x_{r}, t_{s}\right), \\
r, s=0,1, \ldots, N .
\end{gathered}
$$

This is a system of algebraic equations with $(N+1)$ unknowns and $(N+1)$ equations which can be solved by Newton's iteration method to obtain the value of $a_{i j}$ such that $i, j=0 \ldots N$.

In Volterra case, we let $\tilde{v}_{p q}^{(r, s)}$ instead of $v_{p q}$ in (29)

$$
\begin{gathered}
\sum_{i=0}^{N} \sum_{j=0}^{N} a_{i j} T_{i}\left(x_{r}\right) T_{j}\left(t_{s}\right)-\sum_{p=0}^{M} \sum_{q=0}^{M} k_{p q}^{(r, s)} \tilde{v}_{p q}^{(r, s)}=f\left(x_{r}, x_{s}\right), \\
r, s=0,1, \ldots, N
\end{gathered}
$$

where

$$
\tilde{v}_{p q}^{(r, s)}=\int_{-1}^{x_{r}} \int_{-1}^{t_{s}} T_{p}(y) T_{q}(z)\left[u_{N}(y, z)\right]^{p} d y d z
$$

We remind

$$
\left[u_{N}(x, t)\right]^{p}=\sum_{i=0}^{p_{N}} \sum_{j=0}^{p_{N}} d_{i j} T_{i}(x) T_{j}(t),
$$

where $d_{i j}$ is linear or nonlinear combination of $a_{i j}$. Hence, we can calculate easily $v_{p q}$ and $\tilde{v}_{p q}^{(r, s)}$ by (17) and (21).

Remark 3. In case $F(u(x, t))$ is strongly nonlinear, the Taylor series can be used to approximate $F(u(x, t))$ as a polynomial in $u(x, t)$. Then the above method can be applied easily for general cases (25) and (26).

\section{Numerical results}

In this section, the illustrate examples are given to show efficiency the method proposed in Sections 3 and 4. All of the computations have been done using the Maple 12 with just 10 digits precision. In this study, our criterion of accuracy is the maximum absolute error in relevant intervals. In the other word, we investigate the value of infinity norm of error functions. 


\subsection{Linear examples}

For the following cases, we let $M=6$ and $n=15$.

Example 1. Consider the following Fredholm integral equation

$$
\begin{aligned}
& u(x, t)-\int_{-1}^{1} \int_{-1}^{1}(z \sin x+t y) u(y, z) d y d z \\
= & x \cos t+\frac{4}{3} \sin x-\left(1+\frac{4}{3} \sin (1)\right) t, \quad x, t \in[-1,1],
\end{aligned}
$$

with exact solution

$$
u(x, t)=x \cos t-t .
$$

By using (18) we obtain approximate solution

$$
\begin{aligned}
u_{N}(x, t)= & 1.000000001 x-1.000000002 t-0.4999999824 x t^{2} \\
& -1.702672594 \times 10^{-8} x^{2} t+\cdots-3.416035551 \times 10^{-7} x^{8} t^{7} \\
& +0.000000000 x^{8} t^{8} .
\end{aligned}
$$

The maximum absolute errors are shown in Table 1 for $N=3,5$ and 8. Also you can see Figure 1(a).

\begin{tabular}{|c|c|c|r|}
\hline Example & $N=3$ & $N=5$ & \multicolumn{1}{|c|}{$N=8$} \\
\hline 1. & $1.1 \times 10^{-2}$ & $8.7 \times 10^{-5}$ & $2.4 \times 10^{-9}$ \\
2. & $4.2 \times 10^{-4}$ & $8.8 \times 10^{-7}$ & $9 \times 10^{-9}$ \\
3. & $1.2 \times 10^{-2}$ & $8.9 \times 10^{-5}$ & $2.1 \times 10^{-8}$ \\
4. & $3.2 \times 10^{-4}$ & $6.4 \times 10^{-7}$ & $8 \times 10^{-10}$ \\
\hline
\end{tabular}

Table 1 - Maximum absolute errors are presented for Example 1, 2, 3 and 4.

Example 2. Consider the following Fredholm integral equation

$$
\begin{aligned}
& u(x, t)-\int_{0}^{1} \int_{0}^{1}\left(x y+t e^{z}\right) u(y, z) d y d z \\
= & x e^{-t}+\left(\frac{1}{3} e^{-1}-\frac{7}{12}\right) x-\frac{1}{2} t \quad x, t \in[0,1],
\end{aligned}
$$


with exact solution $u(x, t)=x e^{-t}+t$. By using (18) we have

$$
\begin{aligned}
u_{N}(x, t)= & 1.000000001 x+0.9999999972 t-0.999995432 x t \\
& +0.4999883358 x t^{2}+\cdots+0.02307911926 x^{8} t^{8} .
\end{aligned}
$$

(a)

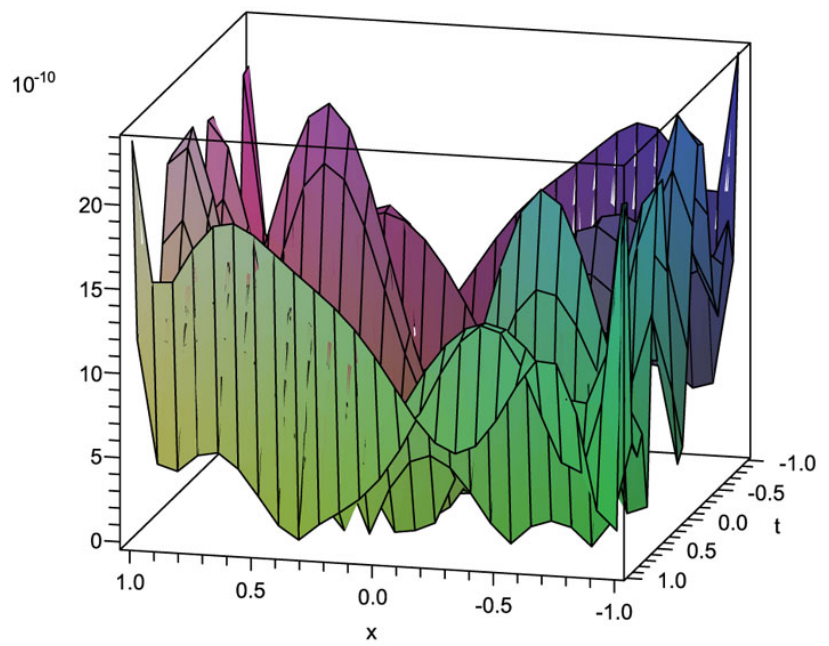

(b)

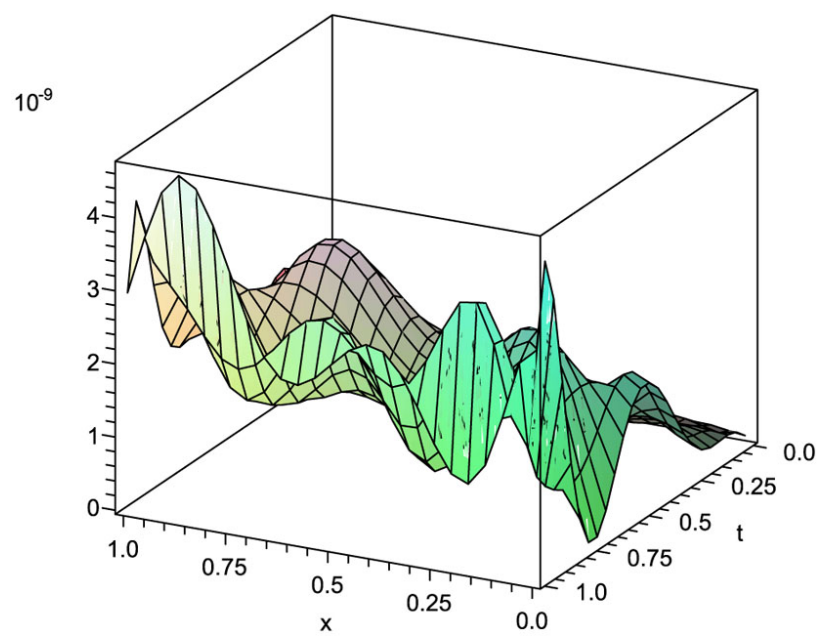

Figure 1 - The error functions of Example 1 and 2 from top to bottom are presented respectively. These examples show the efficiency of the method for Fredholm integral equations. 
The maximum absolute errors are presented in Table 1 for $N=3,5$ and 8 . Also you can see Figure 2(a).

Example 3. Consider the following Volterra integral equation

$$
u(x, t)-\int_{-1}^{t} \int_{-1}^{x}\left(x^{2} y^{2}+z e^{t}\right) u(y, z) d y d z=f(x, t) \quad x, t \in[0,1],
$$

where

$$
f(x, t)=e^{-t}\left(x-\frac{1}{4} x^{2}+\frac{1}{4} x^{6}\right)-\frac{1}{4} e x^{6}+\left(\frac{1}{4} e+\frac{1}{2}\right) x^{2}+\frac{1}{2} x^{2} t-\frac{1}{2} t-\frac{1}{2},
$$

and exact solution is $u(x, t)=x e^{-t}$. By applying (18) and considering (20) we obtain approximate solution as follows

$$
\begin{aligned}
u_{N}(x, t)= & 1.000000000 x-0.9999998304 x t+0.4999999899 x t^{2} \\
& +\cdots-5.678168292 \times 10^{-8} x^{8} t^{8} .
\end{aligned}
$$

The maximum absolute errors are shown in Table 1 for $N=3,5$ and 8. Also you can see Figure 1(a).

Example 4. Consider the following Volterra integral equation [19]

$$
\begin{aligned}
& u(x, t)-\int_{0}^{t} \int_{0}^{x}\left(x y^{2}+\cos z\right) u(y, z) d y d z \\
= & x \sin t-\frac{1}{4} x^{5}+\frac{1}{4} x^{5} \cos t-\frac{1}{4} x^{2}(\sin t)^{2} \quad x, t \in[0,1],
\end{aligned}
$$

with exact solution $u(x, t)=x \sin t$. By applying (18) and considering (20) we obtain approximate solution as follows

$$
\begin{aligned}
u_{N}(x, t)= & .9999999216 x t+0.0000016358 x t^{2}+0.0000013773 x^{2} t \\
& +\cdots+0.0040792670 x^{8} t^{8} .
\end{aligned}
$$

The numerical results are shown in Table 1 are computed errors. Also you can see Figure 2(b).

In Table 1 we investigate the above examples and shows the maximum absolute error. The numerical results shows high accuracy even for small $N$. 
(a)

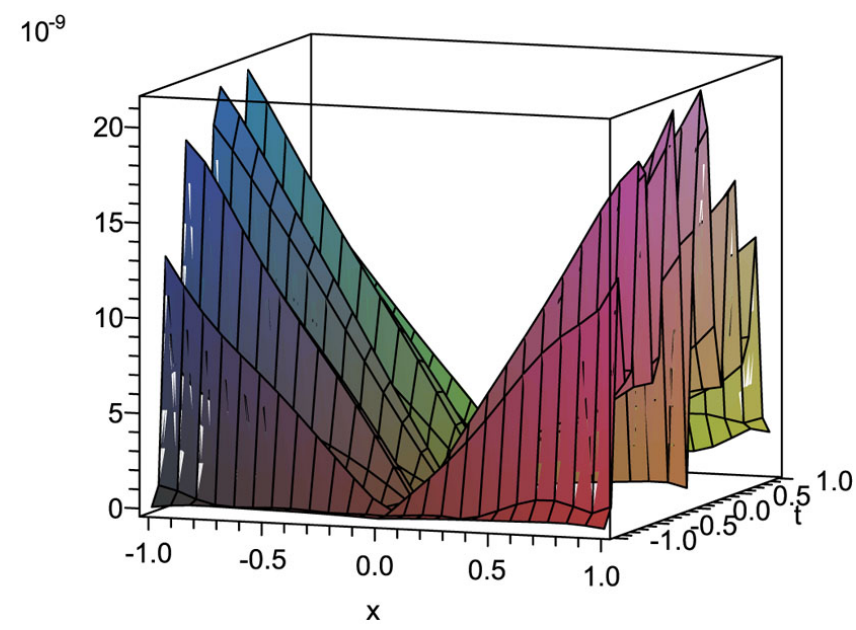

(b)

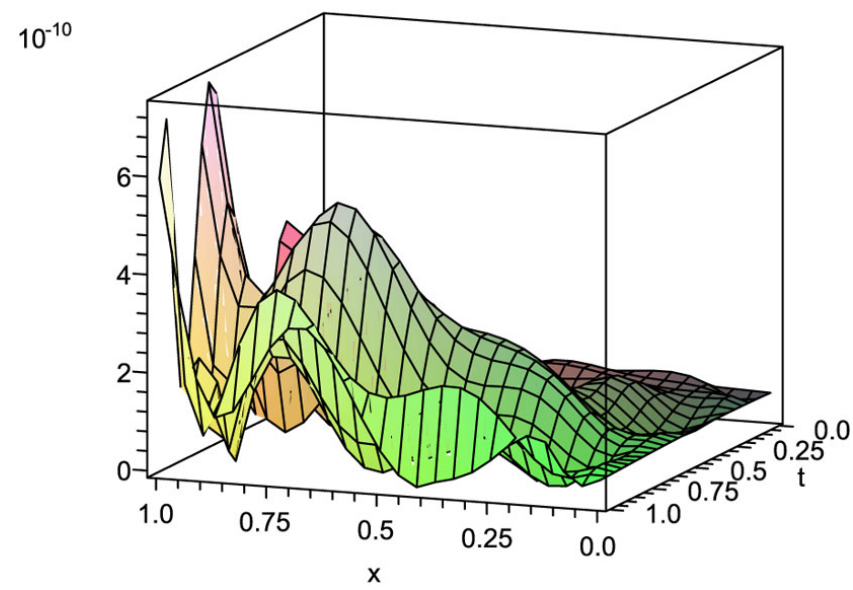

Figure 2 - The error functions of Example 3 and 4 from top to bottom are presented respectively. These examples confirm the efficiency of the method for Volterra integral equations.

Also, comparison between estimated absolute errors of Example 4 for $N=8$ using presented method and differential transform method [19] are illustrated in Table 2. The results show more accuracy and smoother error function by described method. 


\begin{tabular}{|c|c|c|}
\hline$(x, t)$ & $N=8$ (differential transform method) & $N=8$ (presented method) \\
\hline$(.2, .2)$ & $2.820844 \times 10^{-13}$ & $2.727401 \times 10^{-11}$ \\
$(.2, .8)$ & $7.354498 \times 10^{-8}$ & $5.479929 \times 10^{-10}$ \\
$(.4, .6)$ & $1.107230 \times 10^{-8}$ & $2.279254 \times 10^{-12}$ \\
$(.4,1)$ & $1.092336 \times 10^{-6}$ & $3.049593 \times 10^{-10}$ \\
$(.6, .2)$ & $8.462531 \times 10^{-13}$ & $4.405731 \times 10^{-10}$ \\
$(.6, .8)$ & $2.206350 \times 10^{-7}$ & $3.243791 \times 10^{-10}$ \\
$(.8, .4)$ & $5.770791 \times 10^{-10}$ & $7.240839 \times 10^{-10}$ \\
$(.8, .8)$ & $2.941799 \times 10^{-7}$ & $2.585837 \times 10^{-10}$ \\
$(1,6)$ & $2.768074 \times 10^{-8}$ & $2.300421 \times 10^{-10}$ \\
$(1,1)$ & $2.730839 \times 10^{-6}$ & $2.773500 \times 10^{-10}$ \\
\hline
\end{tabular}

Table 2 - Comparison between estimated absolute errors of Example 4 for $N=8$ using presented method and differential transform method [19]. The results show more accuracy for described method.

\subsection{Nonlinear examples}

Example 5. Consider the following Fredholm integral equation

$$
\begin{aligned}
& u(x, t)-\int_{0}^{1} \int_{0}^{1}(y+z)[u(y, z)]^{2} d y d z \\
= & x \cos t-\frac{1}{8}-\frac{7}{24} \cos (1) \sin (1)-\frac{1}{12}[\cos (1)]^{2},
\end{aligned}
$$

where $x, t \in[0,1]$ and exact solution is $u(x, t)=x \cos t$. If we let $M=1$, $N=6$ and $n=15$, by considering (29) we obtain approximate solution as follows

$$
\begin{aligned}
u_{N}(x, t)= & 1.000000000 x-4.895686000 \times 10^{-7} x t-.4999882352 x t^{2} \\
& -\cdots+0.000000000 x^{6} t^{6} .
\end{aligned}
$$

Also, the value of infinity norm of error function is $2.3 \times 10^{-8}$.

Example 6. Consider the following Volterra integral equation [9]

$$
\begin{aligned}
& u(x, t)-\int_{0}^{t} \int_{0}^{x}[u(y, z)]^{2} d y d z \\
= & x^{2}+t^{2}-\frac{1}{45} x t\left(9 x^{4}+10 x^{2} t^{2}+9 t^{4}\right) \quad x, t \in[0,1],
\end{aligned}
$$




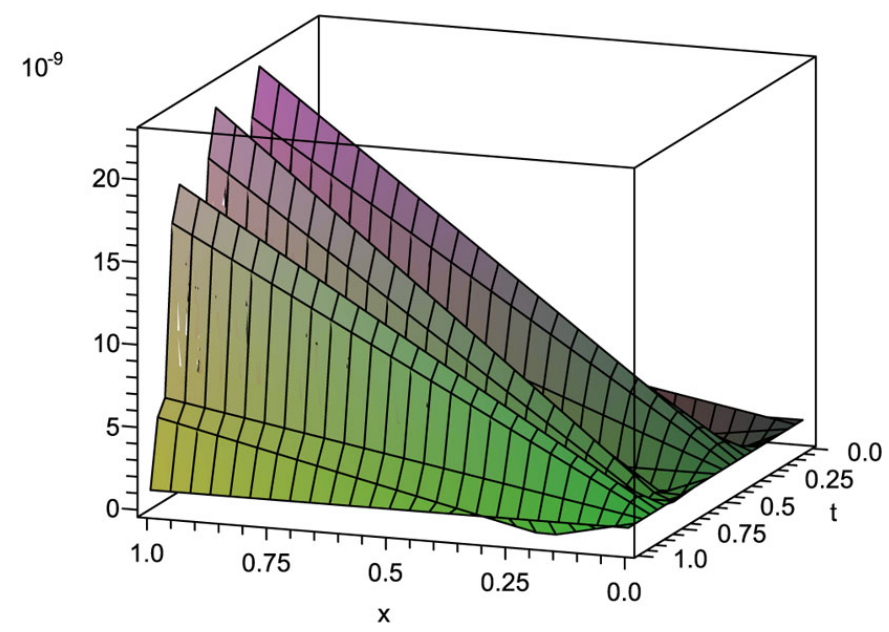

Figure 3 - The error function of Example 5 is shown. This example confirms the efficiency of the method for nonlinear integral equations.

In this case, we let $M=1, N=2$ and $n=15$. By considering (30) and solving the obtained system we have

$$
\begin{array}{ll}
a_{00}=\frac{3}{4}, & a_{01}=\frac{1}{2}, \quad a_{02}=\frac{1}{8}, \\
a_{10}=\frac{1}{2}, & a_{11}=0, \quad a_{12}=0, \\
a_{20}=\frac{1}{8}, & a_{21}=0, \quad a_{22}=0 .
\end{array}
$$

The values of $a_{i j}$ lead to $u(x, t)=x^{2}+t^{2}$ which is the exact solution.

\section{Conclusion}

Analytical solution of the two dimensional integral equations are usually difficult. In many cases, it is required to approximate solutions. In this work, the two dimensional linear and nonlinear integral equations of the second kind are solved by using Chebyshev polynomials through collocation scheme. However this method only works when $F$ is a power function as (27) and (28), we know other cases can be rewritten easily in this form using Taylor extension. The privilege of the method is simplicity and spectrally accuracy $[4,11]$. The illustrative 
examples confirm the validity and efficiency of the method. This method can be extended for the system including such the equations. Also, development of the method can solve the two dimensional integro-differential equations.

\section{REFERENCES}

[1] M.A. Abdou, A.A. Badr and M.B. Soliman, On a method for solving a twodimensional nonlinear integral equation of the second kind. J. Comput. Appl. Math., 235 (2011), 3589-3598.

[2] K.E. Atkinson, The Numerical Solution of Integral Equations of the Second Kind. Cambridge University Press (1997).

[3] H. Brunner, Collocation Methods for Volterra Integral and Related Functional Equations. Cambridge University Press (2004).

[4] C. Canuto, M.Y. Hussaini, A. Quarteroni and T.A. Zang, Spectral Methods: Fundamentals in Single Domains. Springer-Verlag, Berlin, Heidelberg (2006).

[5] A. Chakrabarti and S.C. Martha, Approximate solutions of Fredholm integral equations of the second kind. Appl. Math. Comput., 211 (2009), 459-466.

[6] M.V.K. Chari and S.J. Salon, Numerical Methods in Electromagnetism. Academic Press (2000).

[7] Z. Cheng, Quantum effects of thermal radiation in a Kerr nonlinear blackbody. J. Opt. Soc. Am. B, 19 (2002), 1692-1705.

[8] W.C. Chew, M.S. Tong and B. Hu, Integral Equation Methods for Electromagnetic and Elastic Waves. Morgan \& Claypool (2009).

[9] H. Guoqiang, K. Hayami, K. Sugihara and W. Jiong, Extrapolation method of iterated collocation solution for two-dimensional nonlinear Volterra integral equations. Appl. Math. Comput., 112 (2000), 49-61.

[10] H. Guoqiang and W. Jiong, Extrapolation of Nystrom solution for two dimensional nonlinear Fredholm integral equations. J. Comput. Appl. Math., 134 (2001), 259-268.

[11] B. Fornberg, A Practical Guide to Pseudospectral Methods. Cambridge University Press (1998).

[12] A.J. Jerri, Introduction to Integral Equations with Applications. John Wiley and Sons, INC (1999).

[13] P.K. Kythe and M.R. Schäferkotter, Handbook of Computational Method for Integration. Chapman and Hall/CRC Press (2005). 
[14] Y. Liu and T. Ichiye, Integral equation theories for predicting water structure around molecules. Biophys. Chem., 78 (1999), 97-111.

[15] K. Maleknejad, S. Sohrabi and B. Baranji, Application of 2D-BPFs to nonlinear integral equations. Commun Nonlinear Sci Numer Simulat., 15 (2010), 527-535.

[16] J.C. Mason and D.C. Handscomb, Chebyshev Polynomials. CRC Press LLC (2003).

[17] R. Piesens and M. Branders, Numerical solution of integral equations of Mathematical Physics, using Chebyshev polynomials. J. Comput. Phys., 21 (1976), 178-196.

[18] Q. Tang and D. Waxman, An integral equation describing an asexual population in a changing environment. Nonlinear Analysis, 53 (2003), 683-699.

[19] A. Tari, M.Y. Rahimi, S. Shahmorad and F. Talati, Solving a class of two-dimensional linear and nonlinear Volterra integral equations by the differential transform method. J. Comput. Appl. Math., 228 (2009), 70-76.

[20] K.F. Warnick, Numerical Analysis for Electromagnetic Integral Equations. Artech House (2008).

[21] W.J. Xie and F.R. Lin, A fast numerical solution method for two dimensional Fredholm integral equations of the second kind. Appl. Math. Comput., 59 (2009), 1709-1719. 